\title{
Fatores associados à satisfação das mulheres com a atenção pré-natal em Porto Alegre, Rio Grande do Sul, Brasil
}

\author{
Factors associated with women's satisfaction with prenatal care \\ in Porto Alegre, Rio Grande do Sul, Brazil
}

Janini Cristina Paiz (https://orcid.org/0000-0002-3160-8428) ${ }^{1}$

Patrícia Klarmann Ziegelmann (https://orcid.org/0000-0002-2851-2011) ${ }^{1}$

Ana Cláudia Magnus Martins (https://orcid.org/0000-0003-1413-1098) ${ }^{2}$

Elsa Regina Justo Giugliani (https://orcid.org/0000-0001-6569-6473) ${ }^{1}$

Camila Giugliani (https://orcid.org/0000-0002-2652-5214) ${ }^{1}$

${ }^{1}$ Programa de Pós-

Graduação em

Epidemiologia da

Universidade Federal do Rio Grande do Sul. R. Ramiro

Barcelos $24002^{\circ}$ andar Faculdade de Medicina, Campus Saúde. 90035-003 Porto Alegre RS Brasil. janinicpaiz@gmail.com ${ }^{2}$ Programa de PósGraduação em Saúde da Criança e do Adolescente, Universidade Federal do Rio Grande do Sul. Porto Alegre RS Brasil.

\begin{abstract}
This article aims to identify factors associated with full satisfaction with prenatal care in health services in Porto Alegre (RS), Brazil. This is a cross-sectional study with 287 women that attended prenatal care in the state capital. Women were randomly selected at two large maternity hospitals (public and private) and interviewed at their homes around 30 days after delivery, from January to August 2016. Satisfaction was measured by a Likert scale (very satisfied to very unsatisfied). Prevalence ratios (PR) were estimated by Poisson regression with robust variance, using a hierarchical model. Factors associated with greater satisfaction were higher education ( $P R=1.49$; 95\% CI: 1.08-2.06); multiprofessional care ( $P R=1.29$; 95\% CI: 1.00-1.66); receiving information about breastfeeding ( $P R=1.33 ; 95 \%$ $C I$ : 1.05-1.68) and place of delivery $(P R=1.56$; 95\% CI: 1.12-2.17); and women feeling comfortable asking questions and participating in decisions ( $P R=5.17 ; 95 \%$ CI: 1.79-14.96). The findings suggest that prenatal care services that offer multiprofessional care, provide guidance, and make pregnant women feel comfortable asking and deciding about their care may generate greater satisfaction.
\end{abstract}

Key words Prenatal care, Women's health, $\mathrm{Pa}$ tient satisfaction
Resumo $O$ objetivo deste artigo é identificar fatores associados à plena satisfação com a atenção pré-natal em serviços de saúde de Porto Alegre, Rio Grande do Sul. Estudo transversal, com $287 \mathrm{mu}$ lheres que realizaram pré-natal na capital gaúcha. As mulheres foram selecionadas aleatoriamente em duas maternidades de grande porte (pública e privada) e entrevistadas nos seus domicílios, cerca de 30 dias após o parto, entre janeiro e agosto de 2016. A satisfação foi aferida por meio de escala Likert (muito satisfeita a muito insatisfeita). Foram estimadas razões de prevalência $(R P)$ por regressão de Poisson com estimação robusta da variância, utilizando modelo hierarquizado. Os fatores associados à plena satisfação foram: ingresso no ensino superior ( $R P=1,49$; IC95\%:1,082,06); atendimento multiprofissional ( $R P=1,29$; IC95\%:1,00-1,66); recebimento de orientações sobre amamentação $(R P=1,33$; IC95\%:1,05-1,68) e sobre local do parto $(R P=1,56$; IC95\%:1,122,17); e sentimento da mulher de estar à vontade para fazer perguntas e participar das decisões ( $R P=5,17$; IC95\%:1,79-14,96). Os achados sugerem que serviços de pré-natal que oferecem cuidado multiprofissional, que dão orientações, e que oportunizam às gestantes o sentimento de estar à vontade para questionar e decidir sobre seu cuidado, proporcionam maior satisfação.

Palavras-chave Cuidado pré-natal, Saúde da mulher, Satisfação do paciente 


\section{Introdução}

A atenção pré-natal é uma ferramenta importante de vinculação da gestante à rede de serviços de saúde. Quando prestada por profissionais capacitados, tem potencial para reduzir desfechos negativos de saúde materna e infantil. A assistência pré-natal é composta por ações clínicas e educativas e tem como principais objetivos o acompanhamento do desenvolvimento da gestação, a detecção e o manejo de condições que possam afetar o bem-estar da gestante e do feto e o empoderamento da mulher para o autocuidado, o parto e a prática do aleitamento materno $0^{1-3}$.

Tem sido cada vez mais valorizada a experiência das gestantes e parturientes, o que engloba outras questões, muito além da capacidade dos serviços de fornecerem cuidados rotineiros e protocolares. De acordo com a Organização Mundial da Saúde (OMS), uma experiência satisfatória de gestação envolve a manutenção das condições orgânicas e socioculturais da mulher, uma gestação saudável para mãe e filho, a transição eficaz para o trabalho de parto e nascimento e o alcance da experiência de maternidade com autonomia, competência e autoestima ${ }^{4}$.

No contexto brasileiro, o pré-natal é realizado prioritariamente na rede pública. Estudo de abrangência nacional identificou que 74,6\% das gestantes, entre os anos de 2011 e 2012, receberam assistência pelo sistema público, e que 89,6\% dos acompanhamentos foram realizados na Atenção Primária à Saúde (APS) ${ }^{1}$. Alguns aspectos diferenciam a assistência pré-natal realizada na APS da rede pública daquela realizada no setor privado, entre eles a proximidade do serviço ao domicílio, a provisão de cuidados baseada em protocolos clínicos desenvolvidos pelo Ministério da Saúde e o princípio de redução das desigualdades regionais e socioeconômicas na assistência, pressupostos da APS 5 .

A satisfação é um indicador de qualidade da assistência que enfatiza o protagonismo do usuário, levando em conta a percepção deste sobre determinados serviços ou produtos ${ }^{6}$. Ao identificar as mudanças positivas em desfechos de saúde de mães e crianças associadas com a atenção prénatal, diversos autores estudaram a adequação do cuidado prestado às recomendações vigentes, utilizando indicadores diretos de qualidade, tais como: número de consultas, realização de procedimentos e exames padrão, imunização em tempo oportuno, entre outros ${ }^{1,2,7-9}$.

No entanto, até o presente, poucos são os estudos que avaliam satisfação com a atenção pré-natal. Aqueles que se preocupam com essa abordagem são majoritariamente qualitativos, incluem um número pequeno de mulheres e, em geral, avaliam o cuidado prestado à gestante por uma única categoria profissional e são pouco abrangentes quanto ao número e a variedade de serviços estudados ${ }^{10,11}$. Por essas características, acabam não retratando de forma ampla a percepção das mulheres sobre o cuidado recebido.

Assim, este estudo tem como objetivo identificar, por meio da percepção das mulheres, os fatores associados à maior satisfação com a atenção pré-natal, no período de janeiro a agosto de 2016.

\section{Métodos}

\section{Delineamento e população do estudo}

Realizou-se um estudo transversal, com mulheres que fizeram pré-natal nas redes pública e privada de saúde de Porto Alegre, Rio Grande do Sul. As mulheres foram selecionadas aleatoriamente, por sorteio, em duas maternidades de grande porte (uma pública e outra privada, responsáveis, no total, por aproximadamente $25 \%$ dos 30.268 partos ocorridos na capital gaúcha em 2016), independentemente da classificação do risco gestacional.

Foram excluídas do estudo mulheres ou bebês com desfechos desfavoráveis no momento do parto (óbito ou internação em cuidados intensivos), para evitar interferência na aferição da satisfação. Mulheres com alguma contraindicação para amamentar também foram excluídas em função de outros desfechos medidos na pesquisa, relacionados ao aleitamento materno, que não são objeto deste estudo ${ }^{12,13}$. Mulheres residentes em áreas de risco para visita domiciliar também foram excluídas do estudo para preservar a segurança da equipe de pesquisa.

O tamanho da amostra foi calculado no Programa Power Sample. Com uma prevalência de plena satisfação com o pré-natal igual a $64 \%{ }^{14} \mathrm{e}$ erro de estimativa de seis pontos percentuais, $o$ tamanho da amostra calculado foi de 265 (o que totaliza 318 com o acréscimo de $20 \%$ para eventuais perdas).

\section{Coleta de dados}

A coleta de dados ocorreu entre janeiro e agosto de 2016. Diariamente, todas as mulheres que tivessem parto nas 24 horas anteriores e que atendiam aos critérios de inclusão recebiam uma 
numeração, que foi utilizada para sorteio. Eram sorteadas, por dia, duas mulheres na maternidade pública e uma na privada, até alcançar a amostra pretendida. Essa proporção visou garantir uma representatividade razoável em relação à utilização dos serviços públicos e privados, descrita na literatura como aproximadamente $70 \%$ e $30 \%$, respectivamente, no nível nacional ${ }^{15-17}$. No período de 31 a 37 dias após o parto, realizou-se uma entrevista no domicílio ou, raras vezes, em outro local de preferência da mulher, para aplicação de um questionário estruturado, elaborado especificamente para este estudo, com base na experiência pregressa das pesquisadoras e nos documentos orientadores da atenção pré-natal no contexto brasileiro ${ }^{5,18}$. Foram consideradas perdas as mulheres que não foram encontradas para realização da entrevista após pelo menos três tentativas de contato telefônico e uma presencial.

As entrevistas foram realizadas após projeto piloto que apontou necessidade de pequenos ajustes de natureza semântica no questionário. A equipe de campo foi composta por 12 entrevistadoras treinadas.

As variáveis de exposição (fatores preditores) foram divididas em: sociodemográficas (idade, escolaridade, ocupação, cor da pele autorreferida, nível socioeconômico de acordo com a Associação Brasileira de Empresas de Pesquisa ${ }^{19}$ e situação conjugal da mulher); antecedentes obstétricos (gestações prévias e planejamento da gestação em questão); e atenção pré-natal (local de realização, número de consultas, início do acompanhamento, acompanhada pelo parceiro, atendimento multiprofissional, participação em curso ou grupo de gestantes, recebimento de orientações sobre direitos, local do parto, plano de parto e aleitamento materno, busca de informações e sentimento de estar à vontade para fazer questionamentos e participar das decisões, esta última sendo classificada em três categorias: sim, completamente; mais ou menos; e não).

O desfecho - satisfação da mulher com o atendimento no pré-natal - foi aferido ao final do questionário, após o relato de todos os cuidados e orientações realizados na gestação pelos profissionais de saúde, com a pergunta: "Qual a sua satisfação em relação aos cuidados recebidos ao longo da gestação?” A resposta foi avaliada por meio de escala Likert com cinco opções: muito satisfeita, satisfeita, nem satisfeita/nem insatisfeita, insatisfeita e muito insatisfeita. Devido ao alto percentual de respostas nas categorias "satisfeita" e "muito satisfeita" encontrado no estudo, bem como à escolha das pesquisadoras por investigar os aspectos relacionados com o maior grau de satisfação, o desfecho foi considerado de forma binária: plena satisfação (muito satisfeita), "sim" ou "não".

\section{Aspectos estatísticos e éticos}

A associação entre os fatores preditores e o desfecho "plena satisfação" foi avaliada por meio da regressão de Poisson com estimação robusta da variância, que fornece estimativas para razão de prevalências, segundo modelo hierarquizado ${ }^{20,21}$. De acordo com os pressupostos do modelo, os fatores foram hierarquizados conforme nível de proximidade com o desfecho: distal (características sociodemográficas); intermediário (antecedentes obstétricos) e proximal (características do cuidado pré-natal). Tomando a regra de dez eventos por fator, o tamanho amostral calculado (n: 318) permite a inclusão de 13 fatores em um modelo multivariável ${ }^{22}$.

A Figura 1 apresenta a estrutura do modelo multivariável utilizado neste estudo.

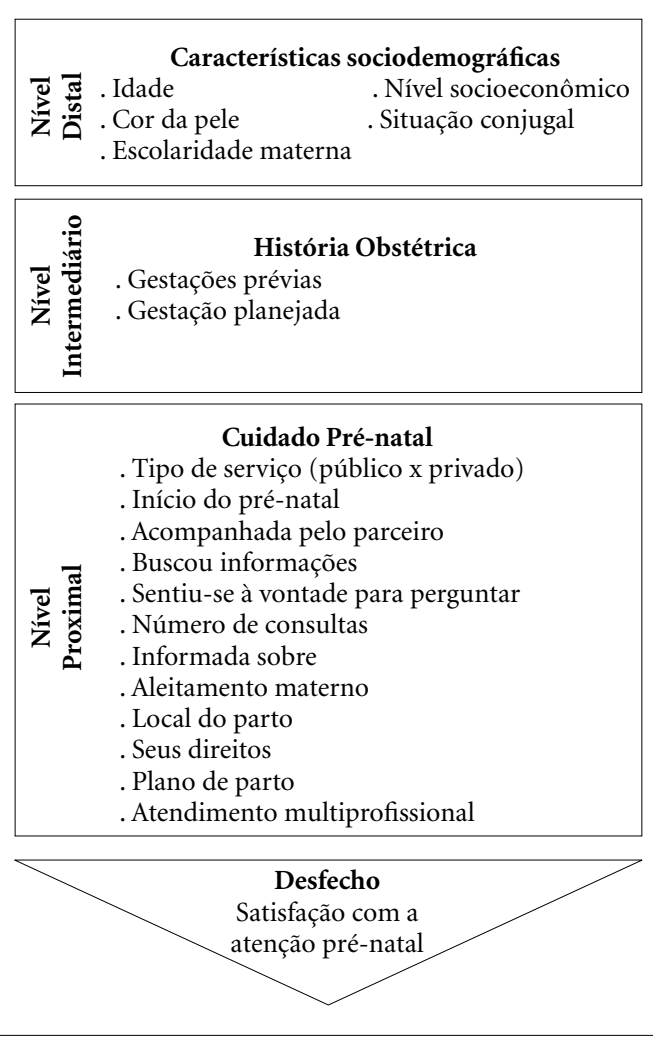

Figura 1. Modelo hierarquizado dos fatores associados à satisfação das mulheres com a atenção pré-natal em Porto Alegre, RS, em 2015-2016.

Fonte: Elaborada pelos autores. 
As estimativas foram ajustadas por todos os fatores que compõem o mesmo nível, independentemente do p-valor. Aqueles fatores que, em seu nível, apresentaram associação com o desfecho, considerando $\mathrm{p}<0,20$, foram incluídos nos níveis seguintes para ajuste de fatores de confusão. No modelo final, o nível de significância adotado foi $\mathrm{p}<0,05$, e os resultados foram expressos em razões de prevalência (RP) com seus respectivos intervalos de confiança (IC) de $95 \%$ e p-valor. O fator idade (contínua) atendeu a suposição de linearidade. A análise estatística foi realizada com auxílio do software SPSS versão 18.0.

Este estudo encontra-se em conformidade com as normas que regem pesquisas com seres humanos $^{23} \mathrm{e}$ foi aprovado pelos Comitês de Ética em Pesquisa das instituições envolvidas. Todas as puérperas que aceitaram participar do estudo assinaram um Termo de Consentimento Livre e Esclarecido (TCLE).

\section{Resultados}

Das mulheres sorteadas neste estudo, 379 foram elegíveis, sendo 287 efetivamente entrevistadas. Houve 25 recusas (6,6\%), e 67 mulheres (17,7\%) foram perdidas por falha no contato para agenda- mento das entrevistas. As mulheres não entrevistadas diferiram em relação à escolaridade e cor da pele, apresentando menor escolaridade (nenhuma mulher havia ingressado no ensino superior versus $43,2 \%$; $\mathrm{p}<0,01$ ) e maior prevalência de cor da pele branca $(87,7 \%$ versus $75,3 \% ; \mathrm{p}=0,032)$ em comparação com aquelas entrevistadas.

A prevalência de plena satisfação na amostra foi de 46,3\% (IC95\%:40,5-50,3). Nas demais categorias, 38,0\% disseram estar satisfeitas, 9,8\% se consideraram nem satisfeitas nem insatisfeitas com o cuidado, 4,9\% estavam insatisfeitas e 1,0\% estava muito insatisfeita com a atenção pré-natal.

A Tabela 1 apresenta as características sociodemográficas, os antecedentes obstétricos e os cuidados recebidos no pré-natal de acordo com a satisfação das mulheres participantes.

A amostra estudada foi composta majoritariamente por mulheres com idade entre 20 e 34 anos, brancas, em relacionamento estável e com ao menos uma gravidez anterior àquela que foi objeto de estudo. Das mulheres plenamente satisfeitas, a maioria pertencia às classes sociais $\mathrm{A}-\mathrm{B}$, tinha ensino superior completo e planejou a gestação, enquanto a maioria das não plenamente satisfeitas pertencia às classes sociais C-D-E, tinha ensino médio completo e não planejou a gestação. (Tabela 1)

Tabela 1. Análise de características sociodemográficas, antecedentes obstétricos e cuidado no pré-natal de acordo com a satisfação das mulheres de Porto Alegre, 2015-2016.

\begin{tabular}{|c|c|c|c|}
\hline \multirow{2}{*}{ Fatores } & \multirow{2}{*}{$\begin{array}{c}\operatorname{Amostra} \mathbf{n}(\%) \\
\mathbf{n}=\mathbf{2 8 7}\end{array}$} & \multicolumn{2}{|c|}{ Plena satisfação n(\%) } \\
\hline & & Sim $133(46,3 \%)$ & Não $154(53,7 \%)$ \\
\hline \multicolumn{4}{|l|}{ Distais: sociodemográficos } \\
\hline \multicolumn{4}{|l|}{ Idade (anos) } \\
\hline Média + DP & $29,1(6,6)$ & $30,3(6,6)$ & $28,0(6,4)$ \\
\hline$\leq 19$ anos & $23(8,0)$ & $8(6,0)$ & $15(9,7)$ \\
\hline 20-34 anos & $199(69,3)$ & $84(63,2)$ & $115(74,7)$ \\
\hline$\geq 35$ anos & $65(22,6)$ & $41(30,8)$ & $24(15,6)$ \\
\hline \multicolumn{4}{|l|}{ Cor da pele } \\
\hline Branca & $216(75,3)$ & $108(81,2)$ & $108(70,1)$ \\
\hline Preta ou parda & $71(24,7)$ & $25(18,8)$ & $46(29,9)$ \\
\hline \multicolumn{4}{|l|}{ Nível socioeconômico* } \\
\hline$A-B$ & $163(57,2)$ & $93(70,5)$ & $70(45,8)$ \\
\hline$C-D-E$ & $122(42,8)$ & $39(29,5)$ & $83(54,2)$ \\
\hline \multicolumn{4}{|l|}{ Escolaridade } \\
\hline Superior (completo ou incompleto) & $124(43,2)$ & $78(58,6)$ & $46(29,9)$ \\
\hline Fundamental e Médio & $163(56,8)$ & $55(41,4)$ & $108(70,1)$ \\
\hline \multicolumn{4}{|l|}{ Situação conjugal $^{*}$} \\
\hline Com companheiro & $188(65,7)$ & $100(75,8)$ & $88(57,1)$ \\
\hline
\end{tabular}


Tabela 1. Análise de características sociodemográficas, antecedentes obstétricos e cuidado no pré-natal de acordo com a satisfação das mulheres de Porto Alegre, 2015-2016.

\begin{tabular}{|c|c|c|c|}
\hline \multirow{2}{*}{ Fatores } & \multirow{2}{*}{$\begin{array}{c}\operatorname{Amostra} \mathbf{n}(\%) \\
\mathbf{n}=\mathbf{2 8 7}\end{array}$} & \multicolumn{2}{|c|}{ Plena satisfação n(\%) } \\
\hline & & Sim $133(46,3 \%)$ & Não $154(53,7 \%)$ \\
\hline \multicolumn{4}{|c|}{ Intermediários: antecedentes obstétricos } \\
\hline \multicolumn{4}{|l|}{ Gestação prévia } \\
\hline Sim & $163(56,8)$ & $69(51,9)$ & $94(61,0)$ \\
\hline \multicolumn{4}{|l|}{ Gestação planejada } \\
\hline Sim & $154(53,7)$ & $83(62,4)$ & $71(46,1)$ \\
\hline \multicolumn{4}{|l|}{ Proximais: atenção pré-natal } \\
\hline \multicolumn{4}{|l|}{ Tipo de serviço } \\
\hline Público & $147(51,2)$ & $50(37,6)$ & $97(63,0)$ \\
\hline Privado ou misto & $140(48,8)$ & $83(62,4)$ & $57(37,0)$ \\
\hline \multicolumn{4}{|l|}{ Idade gestacional de início ${ }^{*}$} \\
\hline$\leq 6$ semanas $^{\star *}$ & $102(36,4)$ & $58(45,0)$ & $44(29,1)$ \\
\hline 7 semanas ou mais & $178(63,6)$ & $71(55,0)$ & $107(70,9)$ \\
\hline \multicolumn{4}{|l|}{ Número de consultas ${ }^{\star}$} \\
\hline$\leq 7$ consultas $^{\star *}$ & $46(16,3)$ & $13(10,0)$ & $33(21,7)$ \\
\hline 8 ou mais & $236(83,7)$ & $117(90,0)$ & $119(78,3)$ \\
\hline \multicolumn{4}{|l|}{ Atendimento multiprofissional ${ }^{*}$} \\
\hline Sim & $62(21,7)$ & $43(32,3)$ & $19(12,4)$ \\
\hline \multicolumn{4}{|l|}{ Buscou informações } \\
\hline Sim & $221(77,0)$ & $109(82,0)$ & $112(72,7)$ \\
\hline \multicolumn{4}{|l|}{ Acompanhada pelo parceiro } \\
\hline Sim, ao menos em uma consulta & $218(76,0)$ & $115(86,5)$ & $103(66,9)$ \\
\hline \multicolumn{4}{|l|}{ Informada sobre seus direitos ${ }^{*}$} \\
\hline Sim, plenamente & $152(53,9)$ & $80(61,5)$ & $72(47,4)$ \\
\hline \multicolumn{4}{|l|}{ Informada sobre local do parto* } \\
\hline Sim & $194(67,8)$ & $105(78,9)$ & $89(58,2)$ \\
\hline \multicolumn{4}{|l|}{ Informada sobre aleitamento materno ${ }^{*}$} \\
\hline Sim, plenamente & $88(30,9)$ & $55(42,0)$ & $33(21,4)$ \\
\hline Não ou parcialmente & $197(69,1)$ & $76(58,0)$ & $121(78,6)$ \\
\hline \multicolumn{4}{|l|}{ Participou de grupo/curso de gestante } \\
\hline Sim & $52(18,1)$ & $27(20,3)$ & $25(16,2)$ \\
\hline \multicolumn{4}{|l|}{ Informada sobre plano de parto ${ }^{*}$} \\
\hline Sim & $37(13,2)$ & $23(17,7)$ & $14(9,3)$ \\
\hline \multicolumn{4}{|l|}{ Sentiu-se à vontade para perguntar } \\
\hline Sim, plenamente & $241(84,0)$ & $130(97,7)$ & $111(72,1)$ \\
\hline Não ou parcialmente & $46(16,0)$ & $3(2,3)$ & $43(27,9)$ \\
\hline
\end{tabular}

Fonte: Elaborada pelos autores.

Quanto à atenção pré-natal, observa-se um elevado número de mulheres que realizou a primeira consulta até a sexta semana de gestação $(36,4 \%)$ e que teve um número total de consultas maior do que oito $(83,7 \%)$. Apenas $21,7 \%$ das mulheres foram atendidas por outros profissionais além daquele responsável pelo pré-natal (médico generalista, ginecologista-obstetra, mé- dico de família e comunidade ou enfermeiro). A presença de acompanhante em ao menos uma consulta de pré-natal ocorreu com $76 \%$ das gestantes. A maioria das mulheres (84\%) afirmou sentir-se plenamente à vontade para fazer perguntas. Quanto ao recebimento de orientações/ informações, $67,8 \%$ foram orientadas sobre o local de realização do parto, $53,9 \%$ sobre seus di- 
reitos, 30,9\% sentiram-se plenamente orientadas sobre aleitamento materno e $13,2 \%$ receberam informações sobre plano de parto. (Tabela 1)

A Tabela 2 apresenta a análise multivariável para identificação dos fatores associados à satisfação das mulheres com o cuidado no pré-natal, segundo modelo hierarquizado.

No nível distal, apenas a escolaridade da mulher mostrou-se associada à satisfação com a atenção pré-natal após ajuste. $O$ ingresso no ensino superior, mesmo que sem conclusão do curso, esteve associado à plena satisfação $(\mathrm{RP}=1,49$; IC95\%:1,08-2,06; $p=0,015)$. Os fatores intermediários não apresentaram significância estatística na análise ajustada. (Tabela 2)

No nível proximal, quatro fatores se mostraram associados com plena satisfação: ter recebido atendimento multiprofissional $(\mathrm{RP}=1,29$; IC95\%:1,00-1,66; $\mathrm{p}=0,049)$; ter sido informada sobre local do parto ( $\mathrm{RP}=1,56$; IC95\%:1,12-2,17; $\mathrm{p}=0,008)$; ter recebido orientações sobre aleitamento materno ( $\mathrm{RP}=1,33$; IC95\%:1,05-1,68; $\mathrm{p}=0,017)$; e sentir-se à vontade para fazer per- guntas ( $\mathrm{RP}=5,17$; IC95\%:1,79-14,96; $\mathrm{p}=0,002)$. Dos profissionais que compartilharam o cuidado das gestantes com os pré-natalistas, $50 \%$ foram de especialidades médicas (endocrinologia e psiquiatria, prioritariamente), $43,5 \%$ de outras profissões da área da saúde (nutricionistas, psicólogos, dentistas e/ou fisioterapeutas) e 6,5\% tiveram atendimento com ambas anteriores.

\section{Discussão}

Este estudo identificou cinco fatores associados à plena satisfação com a atenção pré-natal: alta escolaridade (ingresso no ensino superior), atendimento por equipe multiprofissional, recebimento de orientações sobre aleitamento materno e sobre local do parto e sentimento da mulher de estar à vontade para fazer perguntas e participar das decisões nas consultas.

A satisfação com a assistência pré-natal, segundo a literatura, varia de acordo com a organização dos serviços e dos quesitos avaliados

Tabela 2. Análise multivariável dos fatores associados com a satisfação das mulheres com o cuidado no pré-natal, segundo modelo hierarquizado, em Porto Alegre, 2015-2016.

\begin{tabular}{|c|c|c|c|c|}
\hline Fatores & $\begin{array}{c}\text { Plena Satisfação } \\
\text { n (\%) }\end{array}$ & $\begin{array}{c}\mathrm{RP}_{\mathrm{B}} \\
(\mathrm{IC} 95 \%)\end{array}$ & $\begin{array}{c}\mathbf{R P}_{\mathrm{A}}^{*} \\
(\mathrm{IC} 95 \%)\end{array}$ & P-valor \\
\hline \multicolumn{5}{|l|}{ Distais: sociodemográficos } \\
\hline \multicolumn{5}{|l|}{ Idade (anos) } \\
\hline Média + DP & $30,3(6,6)$ & $1,03(1,01-1,05)$ & $1,00(0,97-1,02)$ & 0,970 \\
\hline \multicolumn{5}{|l|}{ Cor da pele } \\
\hline Branca & $108(50,0)$ & $1,42(1,01-2,00)$ & $1,09(0,77-1,55)$ & 0,596 \\
\hline Preta ou parda & $25(35,2)$ & 1,00 & 1,00 & \\
\hline \multicolumn{5}{|l|}{ Nível socioeconômico } \\
\hline$A-B$ & $93(57,1)$ & $1,78(1,33-2,39)$ & $1,25(0,87-1,82)$ & 0,220 \\
\hline$C-D-E$ & $39(32,0)$ & 1,00 & 1,00 & \\
\hline \multicolumn{5}{|l|}{ Escolaridade } \\
\hline Superior (completo ou incompleto) & $78(62,9)$ & $1,86(1,44-2,40)$ & $1,49(1,08-2,06)$ & 0,015 \\
\hline Fundamental e Médio & $55(33,7)$ & 1,00 & 1,00 & \\
\hline \multicolumn{5}{|l|}{ Situação conjugal } \\
\hline Com companheiro & $100(53,2)$ & $1,63(1,19-2,23)$ & $1,31(0,92-1,86)$ & 0,130 \\
\hline Sem companheiro & $32(32,7)$ & 1,00 & 1,00 & \\
\hline \multicolumn{5}{|l|}{ Intermediárias: antecedentes obstétricos } \\
\hline \multicolumn{5}{|l|}{ Gestação prévia } \\
\hline Sim & $69(42,3)$ & $0,82(0,64-1,05)$ & $0,90(0,71-1,15)$ & 0,418 \\
\hline Não & $64(51,6)$ & 1,00 & 1,00 & \\
\hline \multicolumn{5}{|l|}{ Gestação planejada } \\
\hline $\operatorname{Sim}$ & $83(53,9)$ & $1,43(1,10-1,86)$ & $1,16(0,88-1,53)$ & 0,283 \\
\hline Não & $50(37,6)$ & 1,00 & 1,00 & \\
\hline
\end{tabular}


Tabela 2. Análise multivariável dos fatores associados com a satisfação das mulheres com o cuidado no pré-natal, segundo modelo hierarquizado, em Porto Alegre, 2015-2016.

\begin{tabular}{|c|c|c|c|c|}
\hline Fatores & $\begin{array}{c}\text { Plena Satisfação } \\
\text { n (\%) }\end{array}$ & $\begin{array}{c}\mathbf{R P}_{\mathrm{B}} \\
(\mathrm{IC} 95 \%)\end{array}$ & $\begin{array}{c}\mathbf{R P}_{\mathrm{A}}^{*} \\
(\mathrm{IC} 95 \%)\end{array}$ & P-valor \\
\hline \multicolumn{5}{|c|}{ Proximais: atenção pré-natal } \\
\hline \multicolumn{5}{|l|}{ Tipo de serviço } \\
\hline Público & $50(34,0)$ & $0,57(0,44-0,74)$ & $1,02(0,72-1,45)$ & 0,904 \\
\hline Privado ou misto & $83(59,3)$ & 1,00 & 1,00 & \\
\hline \multicolumn{5}{|l|}{ Idade gestacional } \\
\hline$\leq 6$ semanas & $58(56,9)$ & $1,42(1,11-1,82)$ & $1,05(0,83-1,34)$ & 0,649 \\
\hline 7 semanas ou mais & $71(39,9)$ & 1,00 & 1,00 & \\
\hline \multicolumn{5}{|l|}{ Número de consultas } \\
\hline 8 ou mais & $117(49,6)$ & $1,75(1,09-2,83)$ & $1,12(0,69-1,82)$ & 0,643 \\
\hline$\leq 7$ consultas & $13(28,3)$ & 1,00 & 1,00 & \\
\hline \multicolumn{5}{|c|}{ Atendimento multiprofissional } \\
\hline Sim & $43(69,4)$ & $1,72(1,37-2,17)$ & $1,29(1,00-1,66)$ & 0,049 \\
\hline Não & $90(40,2)$ & 1,00 & 1,00 & \\
\hline \multicolumn{5}{|l|}{ Buscou informações } \\
\hline Sim & $109(49,3)$ & $1,35(0,96-1,91)$ & $1,27(0,87-1,85)$ & 0,201 \\
\hline Não & $24(36,4)$ & 1,00 & 1,00 & \\
\hline \multicolumn{5}{|l|}{ Acompanhada pelo parceiro } \\
\hline Sim & $115(52,8)$ & $2,02(1,33-3,06)$ & $1,37(0,89-2,10)$ & 0,143 \\
\hline Não & $18(26,1)$ & 1,00 & 1,00 & \\
\hline \multicolumn{5}{|l|}{ Informada sobre direitos } \\
\hline Sim, plenamente & $80(52,6)$ & $1,36(1,05-1,78)$ & $1,07(0,83-1,39)$ & 0,577 \\
\hline Não & $50(38,5)$ & 1,00 & 1,00 & \\
\hline \multicolumn{5}{|c|}{ Informada sobre local do parto } \\
\hline Sim & $105(54,1)$ & $1,78(1,27-2,48)$ & $1,56(1,12-2,17)$ & 0,008 \\
\hline Não & $28(30,4)$ & 1,00 & 1,00 & \\
\hline \multicolumn{5}{|c|}{ Informada sobre aleitamento materno } \\
\hline Sim, plenamente & $55(62,5)$ & $1,62(1,27-2,04)$ & $1,33(1,05-1,68)$ & 0,017 \\
\hline Não ou parcialmente & $76(38,6)$ & 1,00 & 1,00 & \\
\hline \multicolumn{5}{|c|}{ Informada sobre plano de parto } \\
\hline Sim & $23(62,2)$ & $2,10(1,03-4,28)$ & $0,89(0,69-1,15)$ & 0,378 \\
\hline Não & $107(43,9)$ & 1,00 & 1,00 & \\
\hline \multicolumn{5}{|c|}{ Sentiu-se à vontade para perguntar } \\
\hline Sim, plenamente & $130(53,9)$ & $8,29(2,75-24,85)$ & $5,17(1,79-14,96)$ & 0,002 \\
\hline Não ou parcialmente & $3(6,5)$ & 1,00 & 1,00 & \\
\hline
\end{tabular}

(estrutura, recursos humanos, disponibilidade de insumos e exames $)^{24}$. Pesquisa desenvolvida no Nordeste brasileiro mostrou que $59,6 \%$ das mulheres estavam satisfeitas com os cuidados recebidos $^{24}$. Proporção muito similar foi encontrada em um município do Sudeste brasileiro, em que $58,8 \%$ das mulheres disseram estar satisfeitas com a assistência ${ }^{25}$. O presente estudo, desenvol- 
milar à demonstrada em âmbito internacional ${ }^{26}$. Não foram encontradas pesquisas, no contexto brasileiro ou no âmbito internacional, com avaliação do desfecho "plena satisfação".

A preocupação em investigar os fatores associados à plena satisfação condizem com as recomendações nacionais e internacionais que visam proporcionar à mulher uma experiência positiva de gestação e maternidade. A atenção no ciclo gravídico-puerperal, costumeiramente avaliada por meio das ações e serviços realizados (número de consultas, exames e procedimentos), pode ser vista de outros ângulos, que valorizem a percepção da mulher e inspirem a busca de novos conceitos e práticas no pré-natal. $\mathrm{O}$ desafio de proporcionar à mulher a plena satisfação com os cuidados deve constituir um dos objetivos de qualidade do serviço de saúde, lado a lado com a execução de técnicas e procedimentos necessários para uma gravidez segura ${ }^{4}$.

Das características sociodemográficas avaliadas, apenas a escolaridade da mulher esteve significativamente associada à satisfação com os cuidados no pré-natal. Mulheres que ingressaram no ensino superior, mesmo que não o tenham concluído, tiveram prevalência $49 \%$ maior de plena satisfação. Pesquisa de avaliação do pré-natal no extremo Sul do Brasil identificou a escolaridade, a renda e a coabitação com companheiro como aspectos associados com a adequação na assistência (número de consultas, início precoce do pré-natal, realização de exames em tempo oportuno, de acordo com Takeda, Coimbra et al. e Silveira et al.), porém esta avaliação não aborda a satifação ${ }^{27}$. Em pesquisa realizada na região $\mathrm{Su}$ deste do Brasil, cujo desfecho foi satisfação com a atenção pré-natal, os autores não identificaram associação de satisfação com renda, escolaridade ou cor da pele da mulher ${ }^{25}$.

Os achados da pesquisa Nascer no Brasil fornecem possíveis explicações para a maior satisfação com a atenção pré-natal entre mulheres com maior escolaridade. Nessa pesquisa, com a ressalva de que foram avaliadas variáveis relativas ao atendimento ao parto, a percepção de menor tempo de espera no atendimento, o tratamento respeitoso por parte dos profissionais de saúde, a privacidade nos exames e no parto, a clareza nas explicações e a possibilidade de fazer perguntas foi significativamente maior em mulheres com elevada escolaridade ${ }^{15}$.

Esses achados ocorrem, possivelmente, porque mulheres com maior escolaridade sentem-se mais seguras e à vontade para questionarem os profissionais, envolverem-se nas decisões sobre seu cuidado, buscarem informações em outras fontes além do profissional que as acompanha, e participarem ativamente no processo de cuidado, recebendo orientações importantes e envolvendo seus parceiros. Vale ressaltar que, na amostra, a proporção de mulheres que ingressaram no ensino superior foi alta $(43,2 \%)$, assim como aquela de estudo realizado no Sul do Brasil, onde 31,1\% das mulheres tinham 12 ou mais anos de estudo ${ }^{28}$.

Esta pesquisa demonstrou que apenas $67,8 \%$ das gestantes foram orientadas sobre o local de realização do parto, 53,9\% sentiram-se plenamente orientadas sobre seus direitos e apenas $30,9 \%$ receberam orientações que julgaram suficientes sobre aleitamento materno. A baixa proporção de mulheres efetivamente orientadas no pré-natal esteve presente em outros estudos nacionais, como em pesquisa realizada na rede de assistência pré-natal do Rio de Janeiro, em que apenas $28,7 \%$ receberam informações sobre o parto e o aleitamento materno ${ }^{29}$.

O empoderamento da mulher, por meio de orientações para o reconhecimento de seus direitos, da segurança e da indicação clínica de cada prática realizada é fundamental para melhorar a experiência de parto e nascimento. Além disso, a assistência pré-natal deve ser um espaço de compartilhamento de informações em relação aos benefícios, técnicas e manejo de situações recorrentes no aleitamento materno. Cabe destacar pesquisa realizada na região Nordeste brasileira, em que $89,8 \%$ das mulheres foram orientadas sobre o aleitamento materno no pré-natal, sendo que o recebimento dessas informações esteve associado com maior prevalência do aleitamento (RP: 5,44; p-valor: 0,003) ${ }^{30}$.

A preocupação dos profissionais com a aplicação de procedimentos de rotina, tais como verificação de tensão arterial, medida de altura uterina e batimentos cardiofetais despende atenção prioritária do pré-natalista, muitas vezes em detrimento de compartilhar orientações importantes com a mulher, que poderiam reforçar o seu conhecimento para vivenciar a gestação e a maternidade com autonomia e confiança ${ }^{4,5}$.

O número de consultas de pré-natal, assim como demonstrado em outras pesquisas, não esteve associado com a satisfação no presente estudo $^{25}$. A qualidade da consulta, em termos de solicitação de exames, realização de procedimentos, esclarecimento de dúvidas e fornecimento de orientações, pode ser um fator que interfira na satisfação, para além do número de atendimentos ${ }^{31}$.

Também não foi encontrada associação entre o local de realização do pré-natal (serviço 
público ou privado/misto) e a satisfação com os cuidados. Pesquisa realizada na Turquia identificou maior satisfação das mulheres que realizaram pré-natal em centros públicos de atenção primária, sendo o acesso geográfico facilitado e a relação interpessoal qualificada os fatores responsáveis pela maior satisfação $0^{32}$. Pesquisas nacionais sobre a temática evidenciam melhores indicadores de qualidade do pré-natal em serviços privados; no entanto, não discutem a satisfação da gestante com o cuidado recebido ${ }^{33}$.

A ausência de diferença no grau de satisfação com a atenção pré-natal em serviços públicos e privados pode estar relacionada com o importante avanço na APS brasileira, em especial na área de saúde materno-infantil, marcado pelo desenvolvimento de ações direcionadas à redução da morbimortalidade, com implementação em larga escala de documentos norteadores e protocolos assistenciais que discutem, além dos procedimentos clínicos de rotina, a importância da humanização do cuidado, do acolhimento e da corresponsabilização da mulher no pré-natal, parto e puerpério ${ }^{5,34,35}$.

Este estudo identificou uma associação positiva entre atendimento multiprofissional e satisfação, achado que é controverso na literatura, apesar de não existirem dados específicos do cuidado pré-natal. Revisão sistemática que incluiu 26 estudos de boa qualidade metodológica, com amostra de 15.526 participantes, demonstrou efeitos positivos da atenção multiprofissional na satisfação do paciente em dez estudos ${ }^{36}$. Porém, no único estudo dessa revisão conduzido em ambiente de atenção à saúde materna, o cuidado multiprofissional não esteve associado à satisfação das mulheres ${ }^{36,37}$.

Os resultados mostram uma importante associação entre satisfação plena com os cuidados no pré-natal e o sentimento de estar à vontade para fazer perguntas e participar das decisões. Esses achados corroboram outras pesquisas nacionais e internacionais, em diferentes cenários de cuidado, que demostram o impacto positivo da relação interpessoal harmoniosa e de respeito entre usuário e profissional na satisfação com os cuidados recebidos ${ }^{10,38}$. Nesse sentido, profissionais que possibilitam à gestante expressar seus medos e inseguranças e construir o processo de maternidade com corresponsabilização, respeito e confiança parecem promover maior satisfação na atenção pré-natal, pois permitem que a mulher esclareça suas dúvidas, conheça seus direitos e atue proativamente nos seus cuidados.

Este estudo apresenta algumas limitações. Variações individuais nas respostas autorreferidas e constrangimento na entrevista face a face podem ter limitado em algum grau a confiabilidade dos resultados. Eventual viés de memória foi minimizado pela realização da entrevista pouco tempo após o parto. A diferença entre as mulheres perdidas $(17,7 \%)$ e aquelas participantes, em termos de escolaridade e cor da pele, pode limitar a utilização do valor encontrado como estimativa da prevalência de plena satisfação, mas não tem influência sobre a verificação das associações. Por fim, a exclusão de mulheres que residem em regiões de extrema violência, bem como o elevado nível socioeconômico e a alta escolaridade da amostra, devem ser considerados como aspectos que possivelmente interferem na capacidade de generalização dos resultados.

Dentre os pontos fortes deste estudo, destaca-se o seu ineditismo, por buscar entender aspectos de qualidade da assistência à saúde ainda pouco explorados no contexto específico do prénatal, com ênfase na satisfação, sempre por meio da percepção das mulheres. Merecem destaque, também, a aleatoriedade da amostra e as entrevistas presenciais realizadas no domicílio, o que diminui a chance de hesitação em criticar o serviço de saúde, minimizando um eventual viés de gratidão. Ressalta-se que a avaliação do desfecho foi feita ao final da entrevista, o que permitiu que a mulher refletisse sobre aspectos específicos do contato com o serviço de saúde antes de avaliá-lo.

\section{Conclusões}

Este estudo evidenciou, de forma inédita, a associação entre plena satisfação com a atenção pré-natal e escolaridade elevada, atendimento multiprofissional e relação profissional-gestante favorável à troca de informações/orientações e empoderamento da mulher. Esses achados representam novos conhecimentos, relevantes e específicos, úteis no contexto das políticas que visam implementar boas práticas no cuidado à gestante e fortalecer as premissas de experiência positiva de gestação, parto e nascimento propostas internacionalmente pela OMS. 


\section{Colaboradores}

JC Paiz respondeu pela concepção e delineamento, análise e interpretação dos dados, redação do artigo e aprovação da versão a ser publicada. PK Ziegelmann fez a análise e interpretação dos dados, revisão crítica do artigo e aprovação da versão a ser publicada. ACM Martins e C Giugliani foram responsáveis pela concepção e delineamento, análise e interpretação dos dados, revisão crítica do artigo e aprovação da versão a ser publicada. ERJ Giugliani respondeu pela concepção e delineamento, revisão crítica do artigo e aprovação da versão a ser publicada.

\section{Referências}

1. Viellas EF, Domingues RMSM, Dias MAB, Gama SGN, Filha MMT, Costa JV, Bastos MH, Leal MC. Assistência pré-natal no Brasil. Cad Saude Publica 2014; 30(Supl.):85-100.

2. Carroli G, Rooney C, Villar J. How effective is antenatal care in preventing maternal mortality and serious morbidity? An overview of the evidence. Paediatr Perinat Epidemiol 2001; 15(Supl. 1):1-42.

3. Barros FC, Bhutta ZA, Batra M, Hansen TN, Victora CG, Rubens CE. Global report on preterm birth and stillbirth (3 of 7): evidence for effectiveness of interventions. BMC Pregnancy Childbirth 2010; 10(Supl. 1):2-36.

4. World Health Organization (WHO). WHO recommendations on antenatal care for a positive pregnancy experience. Geneva: WHO; 2016. 172 p.

5. Brasil. Ministério da Saúde (MS). Protocolos da Atenção Básica: Saúde das Mulheres. Instituto Sírio-Libanês de Ensino e Pesquisa. Brasília: MS; 2016. 230 p.

6. Tzeng HM, Yin CY. Patient satisfaction versus quality. Nurs Ethics 2008; 15(1):121-124.

7. Anversa ETR, Bastos GAN, Nunes LN, Dal Pizzol TS. Qualidade do processo da assistência pré-natal: Unidades Básicas de Saúde e unidades de Estratégia Saúde da Família em município no Sul do Brasil. Cad Saude Publica 2012; 28(4):789-800.

8. Silva EP, Lima RT, Costa MJC, Batista Filho M. Desenvolvimento e aplicação de um novo índice para avaliação do pré-natal. Rev Panam Salud Publica 2013; 33(5):356-362.

9. Doku DT, Neupane S. Survival analysis of the association between antenatal care attendance and neonatal mortality in 57 low- and middle-income countries. Int J Epidemiol 2017; 46(5):1668-1677.

10. Handler A, Rosenberg D, Raube K, Kelley MA. Health care characteristics associated with women's satisfaction with prenatal care. Med Care 1998; 36(5):679694.

11. Barbosa MA, Ferrnandes RÁQ. Avaliação da assistência pré-natal de baixo risco no Município de Francisco Morato-SP. Online Brazilian J Nurs 2008; 7(3):1-14.

12. Bizon AMBL, Giugliani C, Lago JCA, Senna AFK, Martins ACM, Castro SMJ, Giugliani ERJ. Combined pro-breastfeeding practices are advantageous in facilities providing maternity and newborn services. Matern Child Nutr 2019; 15(4):1-8.

13. Senna AFK, Giugliani C, Lago JCA, Bizon AMBL, Martins ACM, Oliveira CAV, Giugliani ERJ. Validation of a tool to evaluate women's satisfaction with maternal breastfeeding for the Brazilian population. J Pediatr 2020; 96(1):84-91.

14. Ribeiro JM, Costa NR, Pinto LFS, Silva PLB. Atenção ao pré-natal na percepção das usuárias do Sistema Único de Saúde: um estudo comparativo. Cad Saude Publica 2004; 20(2):534-545.

15. D’Orsi E, Brüggemann OM, Diniz CSG, Aguiar JM, Gusman CR, Torres JA, Angulo-Tuesta A, Rattner D, Domingues RMSM. Desigualdades sociais e satisfação das mulheres com o atendimento ao parto no Brasil: estudo nacional de base hospitalar. Cad Saude Publica 2014; 30(Supl. 1):154-168. 
16. Paim J, Travassos C, Almeida C, Bahia L, MacInko J. The Brazilian health system: History, advances, and challenges. Lancet 2011; 377(9779):1778-1797.

17. Viacava F, Oliveira RAD, Carvalho CC, Laguardia J, Bellido JG. SUS: Supply, access to and use of health services over the last 30 years. Cien Saude Colet 2018; 23(6):1751-1762.

18. Brasil. Ministério da Saúde (MS). Cadernos de Atenção Básica: atenção ao pré-natal de baixo risco. Brasília:MS; 2012.318 p.

19. Associação Brasileira de Empresas de Pesquisa (ABEP). Critério Brasil 2014. São Paulo: ABEP; 2014. [acessado 2019 out 3]. Disponível em: www.abep.org/ criterio-brasil

20. Boccolini CS, Carvalho ML, Oliveira MIC, Vasconcellos AGG. Factors associated with breastfeeding in the first hour of life. Rev Saude Publica 2011; 45(1):6978.

21. Fuchs SCPC, Victora CG, Fachel J. Modelo hierarquizado: uma proposta de modelagem aplicada à investigação de fatores de risco para diarréia grave. Rev Saude Publica 1996; 30(2):168-178.

22. Harris RJ. A primer of multivariate statistics. $2^{\text {a }}$ ed. New York: Academic Press; 1985.

23. Brasil. Conselho Nacional de Saúde (CNS). Resolução $n^{\circ}$ 466, de 12 de dezembro de 2012. Brasília: CNS; 2012. p. 12. [acessado 2019 out 3]. Disponível em: http:// conselho.saude. gov.br/resolucoes/2012/Reso466.pdf

24. Cardoso MD, Ribeiro CMS, Oliveira IB, Andrade PMC, Santos TMB. Percepção de gestantes sobre a organização do serviço/assistência em um pré-natal de baixo risco de Recife. Revista de Pesquisa Cuidado é Fundamental Online 2016; 8(4):5017-5024.

25. Prudêncio PS, Mamede FV. Avaliação do cuidado prénatal na atenção primária a saúde na percepção da gestante. Rev Gauch Enferm 2018; 39:e20180077.

26. Ostovar R, Mousavizadeh A, Mohamed F, Shahrak G-H, Karimi A. Evaluation of health services provided for women during pregnancy in Yasuj, Iran. J Appl Sciences 2016; 11(11):1416-1421.

27. Cesar JA, Saavedra JS. Uso de diferentes critérios para avaliação da inadequação do pré-natal: um estudo de base populacional no extremo Sul do Brasil. Cad Saude Publica 2015; 31(5):1003-1014.

28. Bertoldi AD, Barros FC, Hallal PRC, Mielke GL, Oliveira PD, Maia MFD, Horta BL, Gonçalves HB, Aluísio JDTRL, Murray J, Victora CG. Tendências e desigualdades em saúde materna e infantil em uma cidade brasileira: metodologia e descrição sociodemográfica de quatro coortes de nascimento de base populacional, 1982-2015. In: Victora CG, Barros FC, Silveira MF, Silva AAF, organizadores. Epidemiologia da desigualdade:quatro décadas de coortes de nascimento. Rio de Janeiro: Fiocruz; 2019. p. 184.

29. Domingues RMSM, Hartz ZMA, Dias MAB, Leal MC Avaliação da adequação da assistência pré-natal na rede SUS do Município do Rio de Janeiro, Brasil. Cad Saude Publica 2012; 28(3):425-437.
30. Tenório MCDS, Mello CS, de Oliveira ACM. Factors associated with the lack of breastfeeding upon discharge from hospital in a public maternity facility in maceió, alagoas, Brazil. Cien Saude Colet 2018; 23(11):3547-3556.

31. Tomasi E, Fernandes PAA, Fischer T, Siqueira FCV, Silveira DS, Thumé E, Duro SMS, Saes MO, Nunes BP,

32. Fassa AG, Facchini LA. Qualidade da atenção prénatal na rede básica de saúde do Brasil: Indicadores e desigualdades sociais. Cad Saude Publica 2017; 33(3):1-11.

33. Erci B, Ivanov L. The relationship between women's satisfaction with prenatal care service and the characteristics of the pregnant women and the service. Eur J Contracept Reprod Heal Care 2004; 9(1):16-28.

34. Cesar JA, Sutil AT, Santos GB, Cunha CF, MendozaSassi RA. Assistência pré-natal nos serviços públicos e privados de saúde: estudo transversal de base populacional em Rio Grande, Rio Grande do Sul, Brasil. Cad Saude Publica 2012; 28(11):2106-2114.

35. Brasil. Ministério da Saúde (MS). Caderno HumanizaSUS: Humanização do parto e do nascimento. $4^{\mathrm{a}}$ ed. Cadernos HumanizaSUS. Brasília: MS;2014. 465 p.

36. Brasil. Ministério da saúde (MS). Humanização do parto: Humanização no pré-natal e nascimento. Brasília: MS;2002. 28 p.

37. Wen J, Schulman KA. Can team-based care improve patient satisfaction? A systematic review of randomized controlled trials. PLoS One 2014; 9(7):1-9.

38. Turnbull D, Holmes A, Shields N, Cheyne H, Twaddle S, Gilmour WH, McGinley M, Reid M, Johnstone I, Geer I, McIlwaine G, Lunan CB. Randomised, controlled trial of efficacy of midwife-managed care. Lancet 1996; 348(9022):213-218.

39. Galle A, Van Parys A-S, Roelens K, Keygnaert I. Expectations and satisfaction with antenatal care among pregnant women with a focus on vulnerable groups: a descriptive study in Ghent. BMC Womens Health $2015 ; 15: 112$

Artigo apresentado em 06/10/2019

Aprovado em 30/05/2020

Versão final apresentada em 01/06/2020

Editores-chefes: Romeu Gomes, Antônio Augusto Moura da Silva 
